\title{
LOW ENERGY IMPROVEMENTS TO THE FERMILAB 400-MEV LINEAR ACCELERATOR
}

\author{
D. E. Young, V. Dudnikov, M. Popovic, C. W. Schmidt and D. Sun \\ Fermi National Accelerator Laboratory *, Batavia, IL 60510, USA
}

\begin{abstract}
Improvements in the Fermilab operating 400-MeV linear accelerator injector are required to achieve the beam intensity and emittance requirement of the Proton Driver design study [5]. It has been determined that these requirements can be achieved by replacing the components in the Linac below $10 \mathrm{MeV}$. An improved $\mathrm{H}^{-}$ion source with an electrostatic transport to a two-section Radio-Frequency Quadrupole (RFQ) accelerator, with the RFQ sections separated by a magnetic five-dimensional phase-space imaging system as used in an earlier Fermilab/SAIC PET Project, and a new 10-MeV drift-tube linac cavity have been studied. It appears possible that an $\mathrm{H}^{-}$intensity of $4.5 \times 10^{13}$ ions per pulse with an improvement in beam emittance from the present system can be achieved with the proposed changes.
\end{abstract}

\section{INTRODUCTION}

The Fermilab Proton Driver Project proposes to replace the Booster accelerator with a rapid-cycling high-intensity 16$\mathrm{GeV}$ synchrotron that would increase the proton beam intensity in the Main Injector by a factor of four. The Linac currently operates with an $\mathrm{H}^{-}$beam current of $50 \mathrm{~mA}$ and a pulse length of $30 \mu \mathrm{sec}$ to give an intensity of $9 \times 10^{12} \mathrm{ppp}$. The required intensity to achieve the Proton Driver goal is an intensity of $3.5 \times 10^{13} \mathrm{ppp}$. Consequently a study was done to determine if the intensity and pulse length in the present Linac could be upgraded by a factor of four to meet the goals of the Proton Driver.

In 1993 the Fermilab Linac was upgraded from an energy of $200 \mathrm{MeV}$ to $400 \mathrm{MeV}$ by replacing the last four drift-tube tanks with side-coupled, 805-MHz modules (CCL) operating at a higher accelerating gradient. The design of the CCL was based on a beam current of $50 \mathrm{~mA}$ and a pulse length less than $100 \mu \mathrm{sec}$. An experiment was performed in 1999 to determine whether the CCL could operate at an intensity required for the Proton Driver design [1]. The ion-source, preaccelerator and drift-tube linac sections were restored to the proton mode of acceleration. It was determined that a beam of nearly $90 \mathrm{~mA}$ at a pulse length of $90 \mu \mathrm{sec}$ could be accelerated to $400 \mathrm{MeV}$, confirming that the CCL could accelerate beams of the required magnitude. The experiment also confirmed that a brighter beam, i.e. higher intensity and smaller emittance, would be desirable. Table 1 shows a consistent set of measurements of the beam emittance as a function of energy along the Linac at a beam intensity of 30 $\mathrm{mA}$. The data show that considerable

* Work supported by the U.S. Department of Energy under contract No. DE-AC02-76CH03000. emittance growth occurs below $10 \mathrm{MeV}$. The emittance dilution in the low energy beam transport systems for $\mathrm{H}^{-}$beams has been widely studied and the RadioFrequency Quadrupole (RFQ) accelerator accepted as the best choice for acceleration of intense beams up to an energy where the drift-tube linac can continue the acceleration. Observe from Table 1 that additional emittance growth occurs in the $10 \mathrm{MeV}$ linac tank. This is understood as misalignments in the quadrupoles in the low energy section of the cavity. The $10 \mathrm{MeV}$ cavity was originally built at Fermilab as a prototype with the expectation that it would be replaced at a later time. By increasing the injection energy into the cavity and improving the alignment of the drift tubes, it is expected that the beam emittance at $10 \mathrm{MeV}$ could be improved.

\begin{tabular}{|c|c|c|c|c|}
\hline & $\begin{array}{c}\text { PREACC } \\
\text { OUT }\end{array}$ & $\begin{array}{c}\text { LINAC } \\
\text { IN }\end{array}$ & $\begin{array}{c}\text { TANK } 1 \\
\text { OUT } \\
\end{array}$ & $\begin{array}{l}\text { LINAC } \\
\text { OUT }\end{array}$ \\
\hline $\begin{array}{l}\text { ENERGY } \\
\text { (MEV) }\end{array}$ & 0.75 & 0.75 & 10 & 400 \\
\hline $\begin{array}{c}\text { INTENSITY } \\
(\mathrm{mA})\end{array}$ & 54 & 49 & 33 & 30 \\
\hline $\begin{array}{l}\text { EMITTANCE } \\
/ \pi \text { (mm-mrad, } \\
95 \%, \text { norm.) }\end{array}$ & 1.3 & 2.6 & 5.2 & 7.8 \\
\hline
\end{tabular}

Table 1 Emittance for $\mathrm{H}^{-}$Beams

It is proposed that the entire low energy end of the present 400-MeV Linac be replaced to include an RFQ and a new $10-\mathrm{MeV}$ drift-tube cavity. In order to maintain the redundancy of a stand-by ion source, it is proposed to divide the RFQ into a low-energy section with its ion source and low-energy beam transport (LEBT) injecting into a second RFQ section to the full RFQ energy. The RFQ sections are connected by a magnetic five-dimensional phase-space imaging system such as that used in the Fermilab/SAIC PET Project [2] and referred to in this study as the double alpha system. The stand-by system with its ion source, LEBT, and low-energy RFQ would include a single alpha magnet and its matching quadrupole magnets. By deactivating the alpha magnet in the failing ion-source system and activating the alpha magnet in the stand-by system, a new ion source can be readily brought online. Separating the RFQ sections also allows ancillary equipment, such as beam chopper, diagnostic equipment, and plasma neutralization devices to be installed in the medium energy transport system (MEBT). A sketch of this proposed low energy system 
with the remaining systems in the linac is shown in Figure 1.

\section{ION SOURCE AND LEBT}

Changing to an RFQ allows the possibility of improving the present $\mathrm{H}^{-}$magnetron ion source or considering other $\mathrm{H}^{-}$ sources to achieve the desired beam intensity and quality. A review of high-intensity negative ion sources for accelerators is given by one of the authors at this conference [3]. It is completely feasible to upgrade the SPS source for the required intensity, duty factor and beam quality without

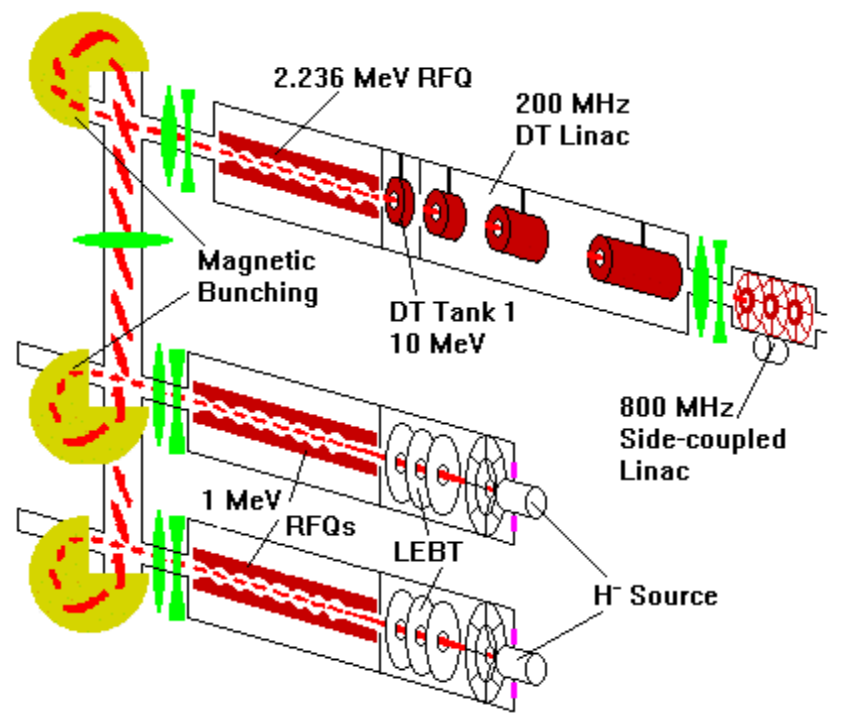

Figure 1. Proposed low-energy improvement sketch.

sacrificing the reliability and availability from its proven past performance. An extraction voltage from the ion source of $50 \mathrm{keV}$ is proposed for extracting sufficient current from the source and allowing for a short electrostatic focusing structure which closely couples the source to the following RFQ. The increased energy of the $\mathrm{H}^{-}$ions allows easier injection and greater transmission through the RFQ.

\section{DOUBLE ALPHA MAGNET MEBT}

Until recently dividing an RFQ into a number of sections to accelerate intense beams was considered unreasonable due to the difficulty of matching the beam between the sections. In the PET Project [2] a beam of ${ }^{3} \mathrm{He}^{+}$ions were accelerated to $1 \mathrm{MeV}$ in a 212-MHz RFQ. At $1 \mathrm{MeV}$ the ions were stripped with a gas jet stripper to form ${ }^{3} \mathrm{He}^{++}$ions which were then focused and injected into a $425-\mathrm{MHz}$ RFQ. The stringent condition of matching the ion beam between the two RFQ sections was done by using a magnetic fivedimensional phase-space imaging system. In this system all particles take the same time to pass through the transport line independent of energy or transverse size and angle so the transverse and longitudinal motions are uncoupled at the entrance to the second RFQ. The performance of this MEBT in the PET Project confirmed the calculations and operated successfully. A similar MEBT using two $270^{\circ}$ dipole magnets and five quadrupoles is proposed in this application.
Using the design code PARMTEQ, RFQs have been designed to accelerate a high intensity, low emittance beam to the entrance of the linac. The first RFQ accepts a 50-keV DC beam from the ion source, bunches it, and accelerates the beam to $1 \mathrm{MeV}$. The second RFQ accelerates the beam from $1 \mathrm{MeV}$ to $2.23 \mathrm{MeV}$. The design allows a beam intensity of $115 \mathrm{~mA}$ to be accelerated with a transmission efficiency of better than $90 \%$. To include the effect of possible emittance growth between the two RFQs, the input emittance of the second RFQ is $20 \%$ larger than the emittance out of the first RFQ.

The code TRACE 3D [4] was used to design the double alpha phase-space imaging system which would transform the $\mathrm{H}^{-}$beam from the match-point at the output of the first RFQ to the match point into the second RFQ. Figure 2 shows the MEBT elements consisting of the two alpha dipoles and five quadrupoles. The apertures of the dipole magnets have been increased a small amount over that used in the PET magnets, but the edge angles have not been altered.

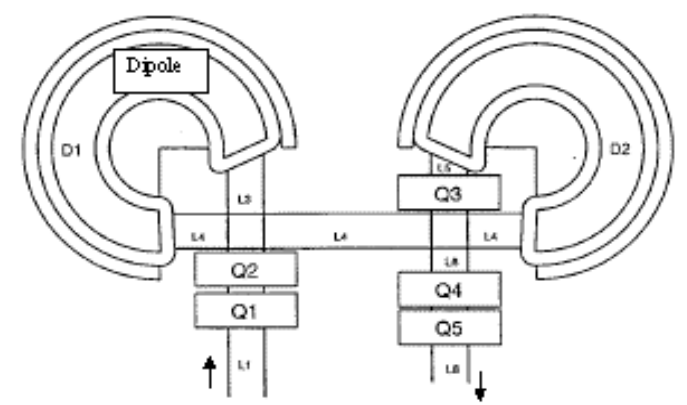

Figure 2. MEBT Elements Consisting of Two Alpha Dipoles and Five Quadrupoles.

Figure 3 shows a good match into the second RFQ for a beam intensity of $80 \mathrm{~mA}$. The small emittance mismatch is well within the acceptance of the RFQ where an emittance growth of $20 \%$ has been allowed. Although this calculation demonstrates the feasibility of using such a system, an R\&D effort will be required to validate the calculations and to derive the parameters for a physical design.

\section{10-MeV DT CAVITY REDESIGN}

By raising the energy into the first cavity of the linac from $0.75 \mathrm{MeV}$ to $2.23 \mathrm{MeV}$ the first 18 drift tubes which have a $2-\mathrm{cm}$ bore diameter and quadrupole lengths of 1 inch and 1 1/4 inch will be eliminated. The new DTL then starts with drift tubes having a $2.5-\mathrm{cm}$ bore and a quadrupole length of 1.75 inches. Thus the problem of misalignments will be significantly mitigated and the cell transit time improved to give a better acceleration rate. The capability of the structure to accelerate higher intensity and better quality beams will be enhanced. 


\section{PARAMETERS AND GOALS}

Table 2 lists the parameters and performance goals of the low-energy improvements to the 400-MeV Fermilab Linear Accelerator to achieve the required intensity of the Proton Driver Project [5].

\section{REFERENCES}

[1] M. Popovic, et.al. "High Current Proton Tests of the
Fermilab Linac," Linac 2000, SLAC, Monterey.

[2] D.J.Larson, et.al. "Ion Optical Design of the BRF-FNALSAIC-UW PET Accelerator," PAC97, Vancouver.

[3] V.Dudnikov. "30 Years of High Intensity Negative Ion Sources for Accelerators," this conference.

[4] K.R. Crandall and D.P. Rusthoi. "Trace 3-d Documentation," LA-UR-97-886.

[5] "The Proton Driver Design Study," Fermilab-TM-2136.

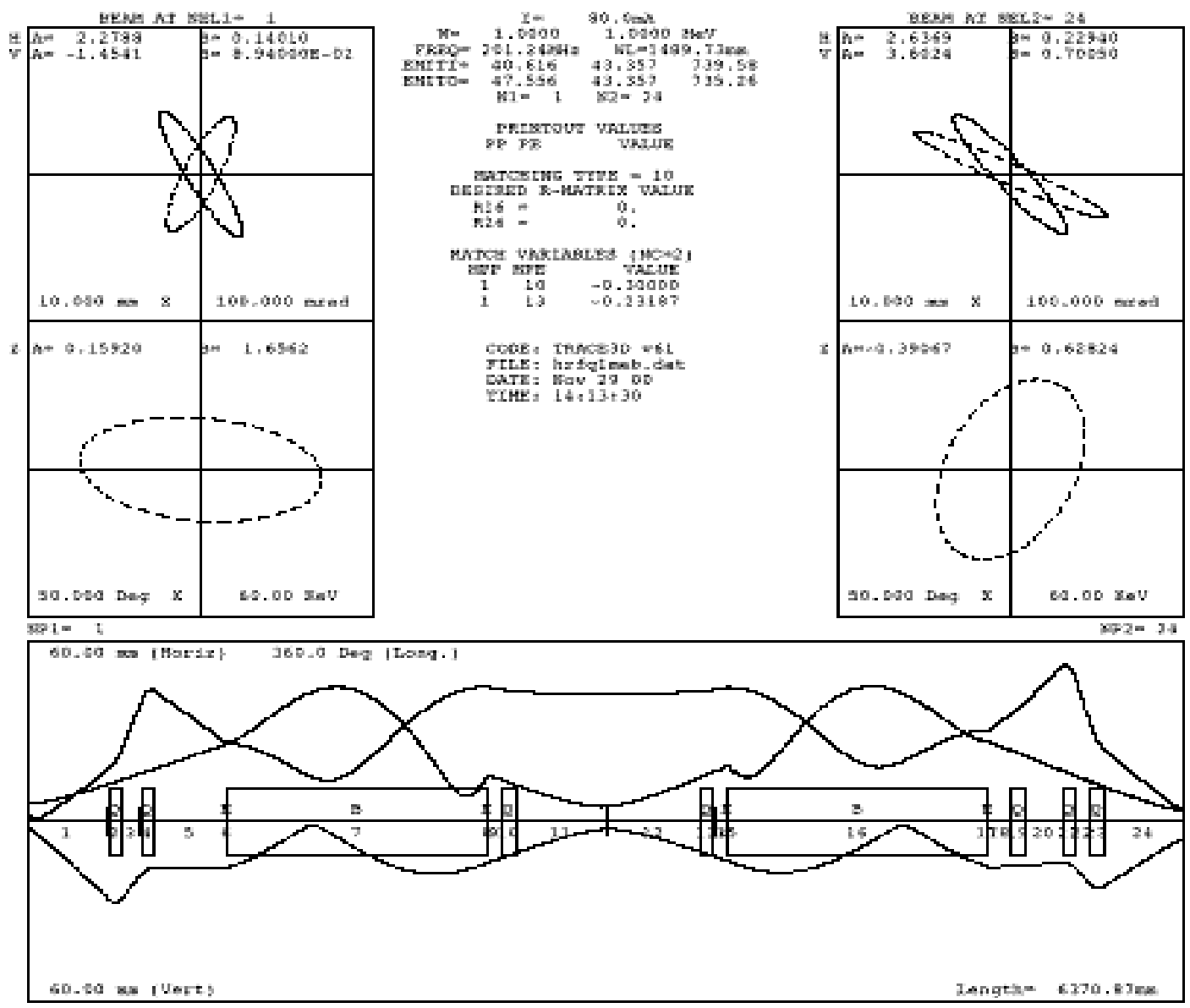

Figure 3. TRACE Calculation Showing Match to RFQ for a Beam Intensity of $80 \mathrm{~mA}$.

\begin{tabular}{|c|c|c|c|c|c|c|c|c|}
\hline & $\begin{array}{c}\text { ION } \\
\text { SOURCE }\end{array}$ & $\begin{array}{c}\text { LEBT/ } \\
\text { CHOPPER }\end{array}$ & RFQ-1 & $\begin{array}{c}540^{\circ} \\
\text { MEBT }\end{array}$ & RFQ-2 & $\begin{array}{c}\text { MATCHING } \\
\text { SECTION }\end{array}$ & DTL & CCL \\
\hline TYPE & $\mathrm{H}$ & $\begin{array}{c}\text { ELECTRO } \\
\text { STATIC } \\
-\end{array}$ & VANE & $\begin{array}{c}\text { DOUBLE } \\
\text { ALPHA }\end{array}$ & VANE & $\begin{array}{c}3 \text { QUADS } \\
1 \text { BUNCHER }\end{array}$ & $\begin{array}{c}\text { DRIFT- } \\
\text { TUBE }\end{array}$ & $\begin{array}{c}\text { COUPLED } \\
- \\
\text { CAVITY }\end{array}$ \\
\hline $\begin{array}{c}\text { OUTPUT ENERGY } \\
(\mathrm{MeV})\end{array}$ & 0.05 & 0.05 & 1 & 1 & 2.23 & 2.23 & 116 & 400 \\
\hline $\begin{array}{c}\text { OUTPUT CURRENT } \\
(\mathrm{mA})\end{array}$ & 115 & 115 & 102 & 102 & 97 & 93 & 86 & 86 \\
\hline $\begin{array}{c}\text { OUTPUT CHOPPED } \\
\text { CURRENT (mA) }\end{array}$ & 115 & 80 & 72 & 72 & 68 & 65 & 60 & 60 \\
\hline $\begin{array}{c}\text { Emittance } \\
(\pi \text { mm-mrad 95\%) }\end{array}$ & 1.2 & 2 & 2.3 & 2.6 & & 2.8 & 3 \\
\hline $\begin{array}{c}\text { FREQUENCY } \\
(\mathrm{MHz})\end{array}$ & 90 & 90 & & 90 & & 90 & 90 \\
\hline $\begin{array}{c}\text { PULSE LENGTH } \\
\text { (msec) }\end{array}$ & & 201 & & 201 & & 90 \\
\hline
\end{tabular}

Table 2. Parameters and Goals for the Performance of the New Configuration of Components for the Low Energy. 